\title{
Dynamical Behaviour of Pressure Fluctuations during Degenerated Combustion Instability
}

\author{
Hiroaki Kobayashi ${ }^{1}$, Hiroshi Gotoda ${ }^{1}$, Shigeru Tachibana ${ }^{2}$ \\ ${ }^{1}$ Department of Mechanical Engineering, Tokyo University of Science \\ 6-3-1 Niijuku, Katsushika, Tokyo, Japan \\ ${ }^{2}$ Aerospace Research and Development Directorate, Japan Aerospace Exploration Agency \\ 7-44-1 Jindaiji-Higashii, Chofu, Tokyo 182-8522, Japan
}

\section{Extended Abstract}

Time series analysis based on the theory of dynamical systems and symbolic dynamics enables us to clarify the deterministic nature hidden in complex phenomena. The potential availability has been studied for various physical settings involving flame front instability induced by swirl/buoyancy coupling [1], double-diffusive instability [2], and a falling film flow instability [3]. Thermoacoustic combustion oscillations arise in a confined combustion system, leading to serious damage in combustors. They possess a wide variety of nonlinear dynamics, and their characterization is of fundamental importance in current combustion science and physics. Thus far, the presence of quasi-periodicity, intermittency, and chaos has been explored for various types of confined turbulent combustors [4-7]. We have also conducted experimental studies [8], [9] on the presence of chaotic dynamics in combustion state close to blowout, but more plausible analysis from different viewpoints is required to show the possible presence of chaos. The aim of this study is to conduct an in-depth analysis to show the possible presence of chaos during degenerated combustion instability [10] in terms of symbolic dynamics and a neural network. We use a lean premixed gas-turbine model combustor [8]. Preheated air and methane are used as premixture in this study. A pressure transducer placing on the wall of combustion chamber acquires pressure fluctuations inside the combustor. We consider the permutation spectrum test [11] consisting of the probability distribution of possible existing rank order patterns in a time series, for distinguishing stochastic and chaotic dynamics. The permutation entropy significantly increases to approximately 0.69 at the equivalence ratio $=0.56$ forming the degenerated combustion instability, which means that the degenerated combustion dynamics possesses high randomness. Zero standard deviation with some forbidden patterns is clearly observed in the permutation spectrum. These results indicate the presence of high-dimensional chaos. In this presentation, we show more detailed results to show the possible presence of chaos in degenerated combustion instability.

\section{References}

[1] H. Gotoda, H. Kobayashi, and K. Hayashi, Physical Review E, vol. 95, p. 022201, 2017.

[2] S. Kondo, H. Gotoda, T. Miyano, and I. T. Tokuda, Physica D, vol. 364, p.1, 2018.

[3] H. Gotoda, M. Pradas, and S. Kalliadasis, Physical Review Fluids, vol. 2, p. 124401, 2017.

[4] L. Kabiraj, A. Saurabh, N. Karimi, A. Sailor, E. Mastorakos, A. P. Dowling, and C. O. Paschereit, Chaos, vol. 25, p. 023101, 2015.

[5] J. Tony, E. A. Goparakrishnan, E. Sreelekha, and R. I. Sujith, Physical Review E, vol. 92, p. 062902, 2015.

[6] S. Balusamy, L. K. B. Li, Z. Han, M. P. Juniper, and S. Hochgreb, Proceedings of the Combustion Institute, vol. 35, p. 3229, 2015.

[7] V. Nair, G. Thampi, and R. I. Sujith, Journal of Fluid Mechanics, vol. 756, p. 470, 2015.

[8] H. Gotoda, H. Nikimoto, T. Miyano, and S. Tachibana, Chaos, vol. 21, p. 013124, 2011.

[9] H. Gotoda, M. Amano, T. Miyano, T. Ikawa, K. Maki, and S. Tachibana, Chaos, vol. 22, p. 043128, 2012.

[10] H. Gotoda, Y. Okuno, K. Hayashi, and S. Tachibana, Physical Review E, vol. 92, p. 052906, 2015.

[11] C. W. Kulp and L. Zunino, Chaos, vol. 24, p. 033116, 2014. 